Old Dominion University

ODU Digital Commons

Electrical \& Computer Engineering Faculty

Publications

Electrical \& Computer Engineering

1982

\title{
Picosecond Laser Pulse Irradiation of Crystalline Silicon
}

K. L. Merkle

H. Baumgart

Old Dominion University, hbaumgar@odu.edu

R.H. Uebbing

F. Phillipp

Follow this and additional works at: https://digitalcommons.odu.edu/ece_fac_pubs

Part of the Engineering Physics Commons, and the Materials Chemistry Commons

\section{Original Publication Citation}

Merkle, K. L., Baumgart, H., Uebbing, R. H., \& Phillipp, F. (1982). Picosecond laser pulse irradiation of crystalline silicon. Applied Physics Letters, 40(8), 729-731. doi:10.1063/1.93207

This Article is brought to you for free and open access by the Electrical \& Computer Engineering at ODU Digital Commons. It has been accepted for inclusion in Electrical \& Computer Engineering Faculty Publications by an authorized administrator of ODU Digital Commons. For more information, please contact digitalcommons@odu.edu. 


\title{
Picosecond laser pulse irradiation of crystalline silicon
}

\author{
K. L. Merkle, ${ }^{\text {a) } H . ~ B a u m g a r t, ~}{ }^{\text {b) }}$ R. H. Uebbing, and F. Phillipp \\ Max-Planck Institut für Metallforschung and Max-Planck Institut für Festkörperforschung, \\ Heisenbergstrasse 1, D-7000 Stuttgart 80, Federal Republic of Germany
}

(Received 17 August 1981; accepted for publication 10 February 1982)

\begin{abstract}
Morphology changes introduced by picosecond laser pulses at $\lambda=532 \mathrm{~nm}$ and $355 \mathrm{~nm}$ in (111) and (100) silicon samples are studied by means of optical and high-voltage electron microscopy. Depending on energy fluence, orientation and wavelength, amorphous or highly defective regions may be created. From an analysis of damage thresholds and damage depth distributions it is concluded that melting and energy confinement precedes the formation of the structural changes.
\end{abstract}

PACS numbers: 79.20.Ds, 81.40.Gh, 78.90. $+\mathrm{t}$

Irradiation of materials with ultrashort laser pulses offers a wide range of possibilities for structural modifications, many of which can be accounted for by the extremely high heating and quench rates offered by this technique.' At present, the mechanism and detailed nature of pulsed laser induced morphology changes in $\mathrm{Si}$ are, however, still subjects of considerable debate. ${ }^{2}$ While most surface modification studies of Si have been devoted to nanosecond pulsed laser annealing of pre-existing amorphous surface layers of $\mathrm{Si}$, it has been reported that an amorphous phase can be formed by irradiating crystalline Si with picosecond laser pulses. ${ }^{3,4}$

The present work is part of a study of the microstructural changes that occur when crystalline $\mathrm{Si}$ is subjected to picosecond laser pulses. The type and spatial arrangement of the damage may yield information on the damage mechanism and on plasma effects, since the pulse duration in the picosecond region would be commensurate to the lifetime of the plasma $\left(\sim 10^{-11} \mathrm{~s}\right) .^{5}$

Single crystalline thin foils $(1-5 \mu \mathrm{m})$ of $(100)$ and (111) orientation were prepared by a large area thinning technique $^{6}$ from $\mathbf{P}$ or B doped $\left(10^{14}-10^{19}\right.$ at $\left./ \mathrm{cm}^{3}\right)$ Si wafers. A passively mode-locked Nd:YAG laser, frequency doubled or tripled, was used to produce pulses of 25-ps full width at half-maximum (FWHM) duration. Single pulses $(\lambda=1.064$ $\mu \mathrm{m}$ ) were frequency doubled in a CDA crystal or tripled by anharmonic mixture of a $\mathrm{KD}^{*} \mathbf{P}$ crystal. A biconvex quartz lens of $10-\mathrm{cm}$ focal length was used to focus the laser pulses to diameters $50-150 \mu \mathrm{m}$ onto bulk Si wafers or prethinned foils. Beam diameters were determined from the out-of-focus position of the sample surface. The ambient atmosphere at the irradiated Si surface was either air or a stream of $\mathrm{Ar}$ gas. The energy of each 25 -ps laser pulse was monitored by diverting a fraction of the intensity via a beam splitter onto a photodiode which had been calibrated against a joule meter. Irradiated bulk Si was used for Nomarski differential interference contrast optical microscopy and for the preparation of cross-sectional samples for electron optical observation. Irradiated thin Si foils were examined optically and by high voltage electron microscopy (HVEM).

The optical and HVEM observations show, for a power level sufficiently above the single pulse damage threshold,

\footnotetext{
a) Visiting Scientist from Materials Science Division, Argonne National Laboratory, Argonne, IL 60439.

b) Present address: Bell Laboratories, Murray Hill, NJ 07974.
}

three distinct regions of different morphology. Figure $1(\mathrm{a})$ illustrates the damage produced by a $1.9 \times 10^{-5} \mathrm{~J}$ laser pulse at $\lambda=355 \mathrm{~nm}$ in (111) $\mathrm{Si}(\sim 5000 \Omega \mathrm{cm}, \mathrm{B}$ doped) viewed by Nomarski differential interference contrast. The outermost bright region (zone 1) corresponds to the amorphous ring observed by Liu et al. ${ }^{3}$ In transmission optical observation, the innermost region (zone 3 ) has approximately the same transparency as the undamaged foil, while zone 1 shows a much reduced transparency, which is still less in zone 2. When the pulse energy is lower, only zones 1 and 2 [Fig. 1(b)] or zone 1 [Fig. 1(c)] alone is observed. HVEM observation of (111) foils indicates a highly defective crystal structure in the region of zone 3 . Figure 2 shows an electron micrograph from this area, taken under dynamical diffraction conditions. The defect contrast is strongly dependent on the orientation of the incident electron beam. In regions of lower defect density within zone 3 , dislocation contrasts and fringe contrasts, possibly from small stacking faults, have been observed. HVEM of zone 2 shows, except for a few isolated

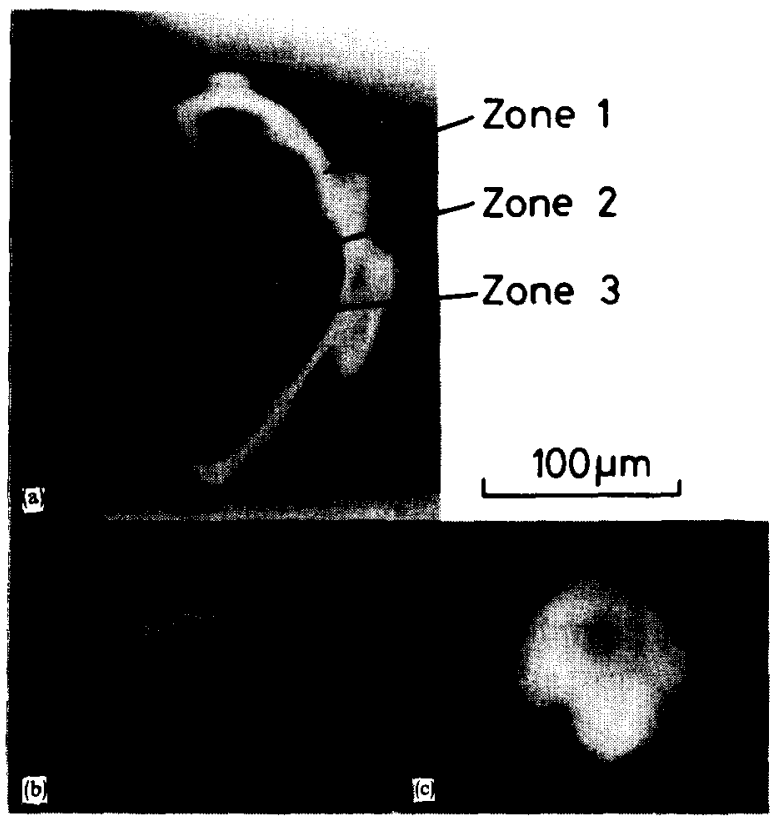

FIG. 1. (111) Si irradiated with single 25-ps laser pulses at $355 \mathrm{~nm}$. Pulse energy ranged between $2 \times 10^{-5} \mathrm{~J}$ and $0.5 \times 10^{-5} \mathrm{~J}$. Zones $1-3,1$ and 2 , and 1 only are visible in (a), (b), and (c), respectively. 


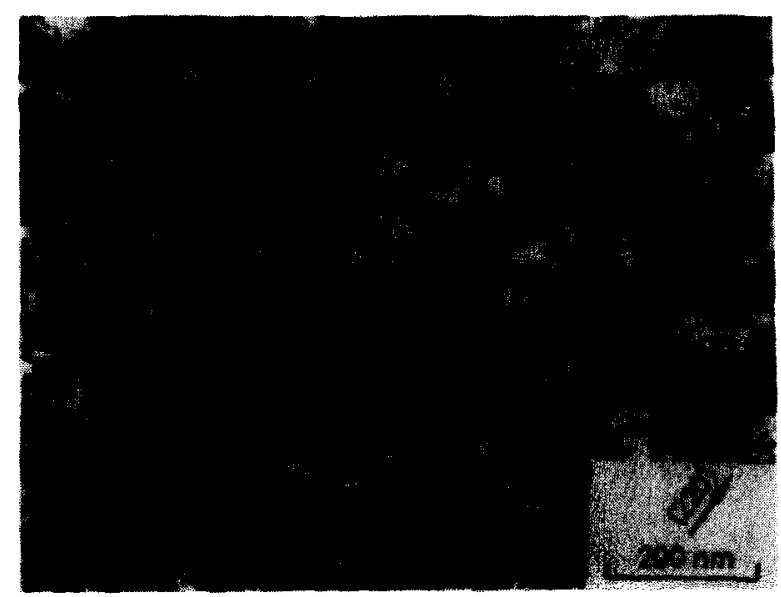

FIG. 2. HVEM micrograph of 25-ps, laser irradiated $(\lambda=355 \mathrm{~nm})(111) \mathrm{Si}$ in zone 3 , near border to zone 2 .

defect clusters near zone 3 , only weak diffraction contrast. Near the border to zone 3 a finely granular modulation is observed in the background intensity. The latter shows throughout zone 2 occasional craterlike features presumably from local regions of higher energy deposition. The contrast behavior in zone 2 is consistent with the presence of a microcrystalline layer, which would also explain the low optical transparency in this range. Across the width of the whole laser spot, the underlying undamaged crystalline foil is strongly bent as indicated by the pronounced excursion of bending contours.

In $(100)$ foils, zone 3 is relatively free of defects and the underlying crystalline substrate is mainly distorted in zone 2 from which typical amorphous rings in the diffraction pattern are obtained. At $\lambda=355 \mathrm{~nm}$, the boundaries of zones 1 and 2 are not well defined in the (100) foils in contrast to the (111) oriented samples.

At $\lambda=532 \mathrm{~nm}$, zone 1 is quite narrow and the boundaries are less sharp in (111) foils, while in (100) foils, zones 1 and 2 were not observed at all at this wavelength. These general features of the damage structures seem not to be dependent on the dopant level and impurity type and whether the laser irradiation was done in air or argon.

The amorphous phase may be quenched in from the liquid when the maximum speed of recrystallization is exceeded. ${ }^{7}$ We presume that, corresponding to the suggestion of Liu et al., ${ }^{3}$ this condition is met in zone 1 . In regions of higher energy fluence, the quenching rates may not be high enough, thus, the Si can revert to the crystalline phase. The recrystallized layers can contain extensive defect structures as seen by HVEM. The differences between the defect morphologies on (100) and (111) surfaces would be consistent with a higher regrowth speed on (100) compared to (111). ${ }^{7.8}$

Whether a melting model is appropriate for the present observations may be judged from examination of the energy deposition parameters. We find that in (111) Si at $\lambda=532$ $\mathrm{nm}$, zone 1 is formed at a total incident laser energy fluence near $0.15 \mathrm{~J} / \mathrm{cm}^{2}$ in a quite narrow energy fluence interval $\left(\Delta \sim 1-2 \times 10^{-2} \mathrm{~J} / \mathrm{cm}^{2}\right)$. Under these conditions the zone 3 threshold lies at $\sim 0.3 \mathrm{~J} / \mathrm{cm}^{2}$. At $\lambda=355 \mathrm{~nm}$ for both (111) and (100) a threshold near $0.1 \mathrm{~J} / \mathrm{cm}^{2}$ is found for the forma-

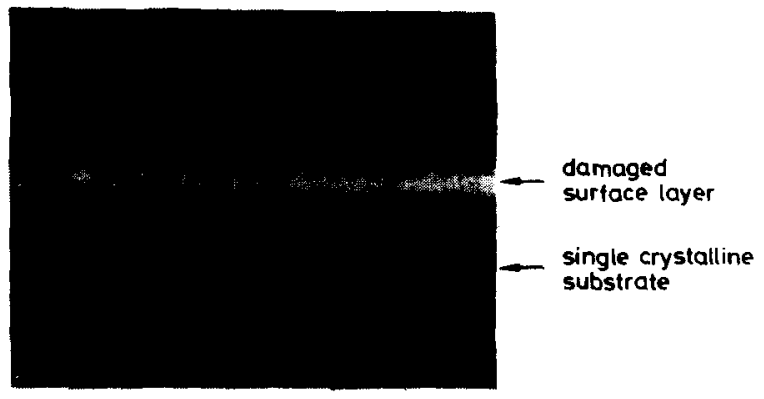

FIG. 3. 25-ps single pulse damage at $\lambda=532 \mathrm{~nm}$. HVEM cross-sectional view in zone $3 ;(220)$ dark field.

tion of zone 1 and a zone 3 threshold of $\sim 0.2 \mathrm{~J} / \mathrm{cm}^{2}$. If the dilution in energy by plasma diffusion and heat transport during the laser pulse is neglected, one obtains for the maximum energy density at the surface reached in a Gaussian beam spot at distance $r$ from the center

$$
\theta_{\max }(r)=\alpha(1-R) E\left(\pi w^{2}\right)^{-1} \exp \left(-r^{2} / w^{2}\right),
$$

where $\alpha^{-1}$ is the absorption depth, $R$ the reflectivity, and $E$ the total energy in the laser pulse.

The energy density required to bring $\mathrm{Si}$ from room temperature to the melting point and melt it is $\theta_{m} \sim 7 \times 10^{3}$ $\mathrm{J} / \mathrm{cm}^{3}$. While at $\lambda=355 \mathrm{~nm}\left(\alpha=10^{6} \mathrm{~cm}^{-1}\right)$ the energy density $\theta_{\max }$ is sufficiently high for melting in all three zones, all zones at $\lambda=532 \mathrm{~nm}\left(\alpha=10^{4} \mathrm{~cm}^{-1}\right)^{9}$ show a $\theta_{\max }$ which falls short of $\theta_{m}$ by a factor of $4-7$, if the room-temperature absorption coefficient is applied.

However, the quite sharp termination of the laser induced structures at the outer edge of zone 1 as well as their sharp limitation in depth as seen in the cross-sectional view of zone 3 in Fig. 3 clearly indicates that melting of a surface layer has taken place in all three zones. Melting can only take place if the absorption depth is strongly decreased during the laser pulse. This could be accomplished (1) if the electronhole plasma directly induces a change in the electronic properties of the solid, as for example a reduction in bandgap ${ }^{10}$ or (2) if $\alpha(1-R)$ strongly increases with temperature and if the carrier-lattice relaxation time is small compared to the pulse length. Liu et al. ${ }^{11}$ recently suggested that a melt threshold of $0.2 \mathrm{~J} / \mathrm{cm}^{2}$ can be fully explained invoking the thermal process alone. However, within the accuracy of the energy deposition parameters and our present knowledge of the temperature dependence of $\alpha$ and $R$, a plasma induced direct contribution to the increased absorption cannot be ruled out. The threshold values and the observed melt depth would for a thermal process alone imply relatively short carrier-lattice relaxation times $\left(\leqslant 5 \times 10^{-12} \mathrm{~s}\right)$.

In view of the above energy considerations, melting can only occur if the energy is more concentrated than indicated by the normal absorption depth ${ }^{9}$ at $\lambda=532 \mathrm{~nm}$ and if the plasma remains localized during the time necessary to transfer most of the energy to the lattice. If the defects were formed in the solid phase by the highly excited plasma itself, one would in contrast to our observations, expect a defect density which is gradually decreasing with depth due to the aforesaid smooth nature of such a process. 
In conclusion, we have demonstrated that energy confinement is necessary to explain the observed defect structures. The damage layer formation is most likely due to a melting and recrystallization process. The high fluence regions do not consist of perfect recrystallized material after picosecond laser irradiation. Details of the defect structures and further implications regarding the damage and plasma annealing model ${ }^{12}$ will be discussed elsewhere.

The authors would like to thank E. Kisela, G. Raithel, and S. Sauter for technical assistance. Part of this work has been supported under the technological program of the Federal Department of Research and Technology (BMFT) of the Federal Republic of Germany. K. L. Merkle would like to acknowledge support from the U. S. Department of Energy.

'N. Bloembergen, in Laser-Solid Interactions and Laser Processing-1978, edited by S. B. Ferris, H. J. Leamy, and J. M. Poate (American Institute of Physics, New York, 1979), p. 1.

${ }^{2}$ See Laser and Electron Beam Processing of Materials, edited by C. W. White and P. S. Peercy (Academic, New York, 1980); Laser and Electron Beam Solid Interactions and Materials Processing, edited by G. F. Gibbons, L. D. Hess, and T. W. Sigmon (North-Holland, Amsterdam, 1981).

${ }^{3}$ P. L. Liv, R. Yen, N. Bloembergen, and R. T. Hodgson, Appl. Phys. Lett. 34, $864(1979)$

${ }^{4} J$. M. Liu, R. Yen, E. P. Donovan, N. Bloembergen, and R. T. Hodgson, Appl. Phys. Lett. 38, 617 (1981).

${ }^{5}$ E. J. Yoffa, Phys. Rev. B 21, 2415 (1980).

'B. O. Kolbesen, K. R. Mayer, and G. E. Schuh, J. Phys. E 8, 197 (1975)

${ }^{7}$ F. Spaepen and D. Turnbull, Conf. Vol., Ref. 1, p. 73.

${ }^{8}$ L. Csepregi, E. F. Kennedy, J. W. Mayer, and T. W. Sigmon, J. Appl. Phys, 49, 3906 (1978).

"W. C. Dash and R. Newman, Phys. Rev. 99, 1151 (1955)

${ }^{10}$ J. A. Van Vechten and M. Wautelet, Phys. Rev. B 23, 5543 (1981).

${ }^{11}$ J. M. Liu, R. Yen, H. Kurz, and N. Bloembergen, Appl. Phys. Lett. 39, 755 (1981).

${ }^{12}$ J. A. Van Vechten, R. Tsu, F. W. Saris, and D. Hoonhout, Phys. Lett.

74A, 417 (1979); J. A. Van Vechten, R. Tsu, and F. W. Saris, Phys. Lett

74A, $422(1979)$

\title{
Growth of InP and InGaAsP $\left(E_{g} \geqslant 1.15 \mathrm{eV}\right)$ layers by liquid phase epitaxy under phosphorus overpressure
}

\author{
V. G. Keramidas, H. Temkin, and W. A. Bonner \\ Bell Laboratories, 600 Mountain Avenue, Murray Hill, New Jersey 07974
}

(Received 18 September 1981; accepted for publication 9 February 1982)

A method is presented for the liquid phase epitaxial growth of stoichiometric InP and InGaAsP $\left(E_{g}>1.15 \mathrm{eV}\right)$ epitaxial layers by creating a phosphorus overpressure in the growth ambient. The effectiveness of the method and the stoichiometry of InP layers can be monitored with the photoluminescent spectra of a phosphorus vacancy-impurity complex.

PACS numbers: $81.10 . \mathrm{Fq}$

The increasing interest in InP-InGaAsP long wavelength emitters and detectors makes essential the preservation of InP surface morphology and stoichiometry throughout the crystal growth and device processing steps. The tendency of InP to thermally decompose because of the disproportionate, preferential evaporation of the high vapor pressure $\mathbf{P},{ }^{\prime}$ causes both structural and stoichiometric changes. ${ }^{2}$ This in turn can affect interfaces of InP with (i) InP and InGaAsP epitaxial layers, (ii) insulating dielectrics, and (iii) ohmic contact metallizations.

Existing studies on thermal degradation of InP and its control have focused on the elimination of macroscopic morphological defects such as those shown in Fig. 1(a). The approaches consist of the use of a phosphine $\left(\mathrm{PH}_{3}\right) / \mathrm{H}_{2} \mathrm{mix}-$ ture ${ }^{3-5}$ the introduction of Ar in the $\mathrm{H}_{2}$ growth ambient ${ }^{6}$ or the use of In-Sn-P solutions. ${ }^{7}$ Surface morphology alone,

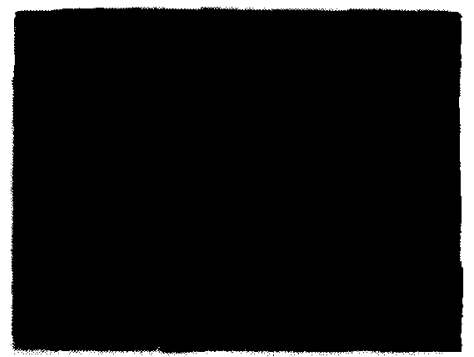

(a)

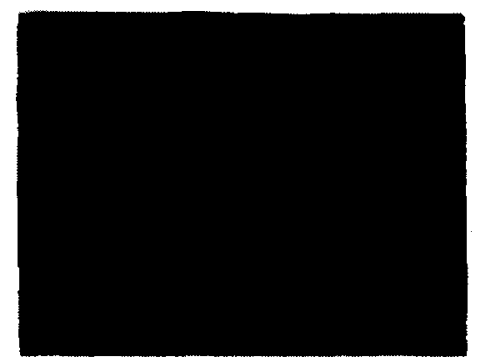

(b)

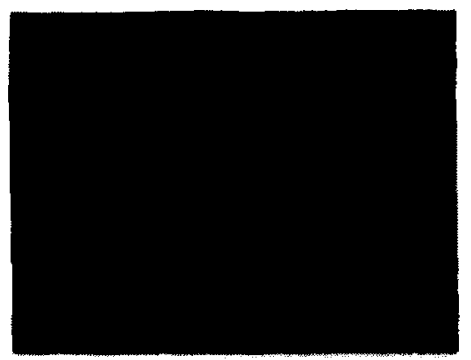

(c)
FIG. 1. (a) Large thermal dissociation features on InP after prolonged exposure to high temperature. (b) Small thermal dissociation features on an InP epilayer after a few seconds exposure to high temperature in pure $\mathrm{H}_{2}$ ambient. (c) Similar layer as (b) grown in $\mathrm{H}_{2}$ ambient with phosphorus overpressure. 